\title{
The Influence of HIV Status on the Burden and Clinical Manifestations of Gastrointestinal Pathogens in Yangon, Myanmar
}

\author{
Win Pa Pa Aung, ${ }^{1} \dagger$ Nan Phyu Sin Toe Myint, ${ }^{2,3,4} \dagger$ Thet Tun Zaw, ${ }^{4}$ David A. Cooper, ${ }^{5} \ddagger$ Ne Myo Aung, ${ }^{2,3,4}$ Mar Mar Kyi, ${ }^{2,3,4}$ \\ Mya Mya Lwin, ${ }^{1,4}$ and Josh Hanson ${ }^{3,4,5 *}$ \\ ${ }^{1}$ Department of Microbiology, University of Medicine 2, Yangon, Yangon, Myanmar; ${ }^{2}$ Insein General Hospital, Yangon, Myanmar; ${ }^{3}$ Department of \\ Medicine, University of Medicine 2, Yangon, Yangon, Myanmar; ${ }^{4}$ Myanmar Australia Research Collaboration for Health (MARCH), Yangon, \\ Myanmar; ${ }^{5}$ The Kirby Institute, University of New South Wales, Sydney, Australia
}

\begin{abstract}
The impact of HIV infection on the burden of gastrointestinal pathogens in Myanmar is poorly defined. Stools of 103 HIV-infected and 105 HIV-uninfected adult outpatients at a tertiary referral hospital in Yangon were examined microscopically. Stool antigen tests for Helicobacter pylori infection were positive in 63/103 (61\%) HIV-infected and $61 / 105(58 \%)$ HIV-uninfected patients $(P=0.65)$. Soil-transmitted helminth infections were much less common, occurring in 9/103 (9\%) HIV-infected and 13/103 (13\%) HIV-uninfected patients $(P=0.50)$. One HIV-uninfected patient had Giardia duodenalis, but there were no cases of Strongyloides stercoralis, Entamoeba histolytica, Capillaria philippinensis, Isospora, Cyclospora, or Schistosoma infection in the entire cohort. Despite the high prevalence of $H$. pylori, only $1 / 208(0.5 \%)$ had ever received eradication, compared with 159/208 (76\%) who had ever been dewormed. Helicobacter pylori appears to be an underappreciated pathogen in Myanmar. Its strong association with gastric cancer and peptic ulcer disease necessitates a more aggressive approach to its management.
\end{abstract}

The few published studies to examine the burden of gastrointestinal parasites in Myanmar report a prevalence that is one of the highest in Asia. ${ }^{1,2} \mathrm{HIV}$ is also an important infection in the country, affecting an estimated $0.8 \%(0.6-0.9 \%)$ of adults aged 15-49 years. ${ }^{3}$ HIV-infected patients in Myanmar frequently present with advanced disease, at which time, the main aim is to initiate life-saving antiretroviral therapy (ART) and to identify life-threatening conditions such as tuberculosis; gastrointestinal pathogens are seen as less of a priority. ${ }^{4}$

However, gastrointestinal parasites are common in HIVinfected patients, and regular screening is an important component of HIV management, ${ }^{5,6}$ especially as the morbidity-and even mortality-from these infections is underestimated. ${ }^{7}$ There are even data to suggest that antihelminthic therapy may have a favorable effect on HIV progression. ${ }^{8}$

The clinical significance of another important gastrointestinal pathogen in Myanmar-Helicobacter pylori-is enormous. The infection's local prevalence approaches $70 \%,{ }^{9}$ and the country's age-standardized death rate for gastric cancer is $11.1 / 100,000 /$ year, while that of peptic ulcer disease is $7.1 /$ $100,000 /$ year. This compares with a rate of $17.4 / 100,000 /$ year for HIV infection. ${ }^{10}$ There are conflicting data about the impact of HIV infection on $H$. pylori colonization, ${ }^{11}$ but it is important to identify any interaction in a country such as Myanmar where both infections are common.

This study determined the prevalence of gastrointestinal parasites and $H$. pylori infection in outpatients at a tertiary referral hospital in Yangon, Myanmar's largest city. It aimed to establish whether a patient's HIV status had any impact on the prevalence of these infections or the symptoms that they caused.

*Address correspondence to Josh Hanson, The Kirby Institute, Level 6, Wallace Wurth Bldg., High St., University of New South Wales, Kensington, New South Wales 2052, Australia. E-mail: jhanson@ kirby.unsw.edu.au

†These authors contributed equally to the work.

$\ddagger$ Professor David Cooper has sadly passed away during the preparation of this report, but contributed significantly to the work.
This cross-sectional study was performed at Insein General Hospital, a 500-bed teaching hospital between March 1 and May 31, 2019. Adults (aged $\geq 18$ years) attending the hospital's ART and general medical outpatient department for any reason were eligible for inclusion. With the study's limited budget, it was planned that data from 100 HIV-infected and 100 unmatched HIV-uninfected patients would be evaluated. Written consent was obtained before enrollment. The only exclusion criterion was the use of antibiotic therapy in the prior 4 weeks which would have vitiated the results of the $H$. pylori stool antigen testing (SAT). Participants completed a dedicated pro forma addressing their demographics, socioeconomic status, and their access to water, sanitation, and hygiene to determine the relative contributions of these factors to the prevalence of gastrointestinal pathogens. The pro forma also asked about current gastrointestinal symptoms, a history of $H$. pylori-related disease, and any prior deworming or $H$. pylori therapy. All patients had their hemoglobin level and mean corpuscular volume (MCV) determined; HIV-infected patients had their most recent CD4 T-cell count recorded and documentation as to whether-or not-they were receiving ART.

The participants were provided with a sealed stool container and asked to provide a specimen. The fresh specimen was tested within hours using a monoclonal antibody-based SAT (BIONEXIA ${ }^{\circledR}$ H. pylori Ag, BioMérieux, Marcy-l'Étoile, France; reported sensitivity: $86.1 \%$ [95\% Cl: 71.3-93.9\%] and specificity: $93.7 \%$ [95\% Cl: $86.9-97.1 \%])^{12}$ by study doctors at the hospital. If participants tested positive, they were treated-according to the hospital policy-with sequential combination therapy of rabeprazole, amoxycillin, clarithromycin, and tinidazole. If repeat SAT was positive, they were offered second-line levofloxacin-based therapy.

Stools were also transported to the microbiology laboratory where experienced microbiologists examined the specimen for the presence of intestinal parasites on the same day. Stools were examined for hookworm, Ascaris lumbricoides, Trichuris trichiura, Strongyloides stercoralis, Giardia duodenalis, Entamoeba histolytica, Capillaria philippinensis, and Cryptosporidium, Microsporidium, Isospora, Cyclospora, and Schistosoma species using the formalin-ether concentration technique 
and modified Ziehl-Neelsen staining, where appropriate. All positive results and $10 \%$ of the negative results were reviewed by a senior microbiologist (W. P. P. A.). All microbiologists were blinded to the HIV status of the patient. Positive stool results were reported to the study doctors who prescribed any necessary treatment.

Data were de-identified, entered into an electronic database, and analyzed with statistical software (Stata 14, StataCorp., College Station, TX). Groups were analyzed using the Kruskal-Wallis and chi-squared tests, where appropriate. The Human Research Ethics Committee of the University of Medicine 2, Myanmar (24/ ERC-1 [10-2017]), provided ethical approval for the study.

The study enrolled 208 patients; 142 (68\%) lived in a city, most commonly in Yangon (98/208, 47\%), while 66 (32\%) lived in a rural area, most commonly the Ayeyarwady delta region (51/ $208,25 \%)$.

There were 105 HIV-uninfected patients and 103 HIVinfected patients, 87 (85\%) of whom were receiving ART. The
HIV-infected patients had a median (interquartile range) CD4 T-cell count of 514 (372-712) cells $/ \mathrm{mm}^{3}, 7 / 103(7 \%)$ had a CD4 T-cell count $<200$ cells $/ \mathrm{mm}^{3}$. Patients with HIV infection were younger and had a lower body mass index than HIVuninfected patients, but did not have a higher prevalence of gastrointestinal pathogens. Indeed, gastrointestinal symptoms were less common among the HIV-infected patients (Table 1).

There were 22/208 (11\%) patients in the cohort with evidence of soil-transmitted helminth (STH) infection; of these, 14 (7\%) were infected with T. trichiura, 5 (2\%) with hookworm, and $3(1 \%)$ with $A$. lumbricoides; one patient was infected with both $T$. trichiura and $A$. lumbricoides (Figure 1). HIV-infected patients were no more likely to have STH infection than HIV-uninfected patients $(9 / 103$ [9\%] versus $13 / 103$ [13\%], $P=0.50$ ), although they were more likely to have been dewormed previously (88/103 [85\%] versus $71 /$ 105 [68\%], $P=0.002)$.

TABLE 1

Demographic, socioeconomic, clinical, and laboratory characteristics of the patients, stratified by HIV status

\begin{tabular}{|c|c|c|c|}
\hline Variable & HIV infected $(n=103)$ & HIV uninfected $^{\star}(n=105)$ & $P$-value \\
\hline \multicolumn{4}{|l|}{ Demographic characteristics } \\
\hline Age (years) & $41(35-49)$ & $58(44-68)$ & 0.0001 \\
\hline Male gender, $n(\%)$ & $44(43)$ & 37 (35) & 0.27 \\
\hline Rural residence, $n(\%)$ & $35(34 \%)$ & $31(30 \%)$ & 0.52 \\
\hline \multicolumn{4}{|l|}{ Socioeconomic characteristics } \\
\hline $\begin{array}{l}\text { Number of people presently living in } \\
\text { household }\end{array}$ & $4(3-5)$ & $4(3-6)$ & 0.03 \\
\hline Number of siblings & $5(3-7)$ & $5(4-7)$ & 0.68 \\
\hline Employed $\dagger$ & $54 / 101(53 \%)$ & $28 / 56(50 \%)$ & 0.68 \\
\hline Completed high school & $42(51 \%)$ & $40(49 \%)$ & 0.70 \\
\hline Private flushing toilet & $21(20 \%)$ & $13(12 \%)$ & 0.12 \\
\hline Drinks purified drinking water & $72(70 \%)$ & 66 (63\%) & 0.28 \\
\hline Municipal water supply & $26(25 \%)$ & $29(28 \%)$ & 0.70 \\
\hline Water from underground aquifer (well) & 75 (73\%) & $71(68 \%)$ & 0.41 \\
\hline Household income $>300,000 \mathrm{MMK} /$ month $\ddagger$ & $27(26 \%)$ & $26(25 \%)$ & 0.81 \\
\hline \multicolumn{4}{|l|}{ Relevant medical history } \\
\hline Ever dewormed & $88(85 \%)$ & $71(68 \%)$ & 0.002 \\
\hline Dewormed last year & $28(27 \%)$ & 17 (16\%) & 0.054 \\
\hline Prior Helicobacter eradication & $1(1 \%)$ & $0(0 \%)$ & 0.50 \\
\hline Active peptic ulcer disease & 0 & 0 & - \\
\hline Past peptic ulcer disease & $1(1 \%)$ & $2(2 \%)$ & 1 \\
\hline Past or present stomach cancer & 0 & 0 & - \\
\hline Family history of stomach cancer & $0(0 \%)$ & $3(3 \%)$ & 0.25 \\
\hline On antiretroviral therapy & $87(84 \%)$ & - & - \\
\hline \multicolumn{4}{|l|}{ Current clinical findings } \\
\hline Current dyspepsia & $21(20 \%)$ & $42(40 \%)$ & 0.002 \\
\hline Current abdominal pain & $11(11 \%)$ & $18(17 \%)$ & 0.18 \\
\hline Current diarrhea & $4(4 \%)$ & $5(5 \%)$ & 1 \\
\hline Current melena & 0 & 1 & 1 \\
\hline Any gastrointestinal symptoms currently & $30(29 \%)$ & $49(47 \%)$ & 0.009 \\
\hline Body mass index $\left(\mathrm{kg} / \mathrm{m}^{2}\right)$ & $21.2(18.9-24.1)$ & $24.2(21.4-27.6)$ & 0.0001 \\
\hline \multicolumn{4}{|l|}{ Laboratory findings } \\
\hline Helicobacter pylori positive & $63(61 \%)$ & $61(58 \%)$ & 0.65 \\
\hline Any parasites & $53(51 \%)$ & $60(57 \%)$ & 0.41 \\
\hline Number of different parasites & $1(0-1)$ & $1(0-1)$ & 0.65 \\
\hline Ascaris lumbricoides & $2(2 \%)$ & $2 / 103(2 \%)$ & 1 \\
\hline Trichuris trichiura & $6(6 \%)$ & $8 / 103(8 \%)$ & 0.78 \\
\hline Hookworm & $2(2 \%)$ & $3 / 103(3 \%)$ & 1 \\
\hline All soil-transmitted helminths & $9(9 \%)$ & $13 / 103(13 \%)$ & 0.50 \\
\hline Microsporidium & $22(21 \%)$ & $15(14 \%)$ & 0.18 \\
\hline Cryptosporidium & $41(40 \%)$ & $48(46 \%)$ & 0.39 \\
\hline Hemoglobin (g/dL) & $12.5(11.5-13.7)$ & $12.4(11.5-13.5)$ & 0.36 \\
\hline Mean corpuscular volume (fL) & $86(79-94)$ & $84(77-87)$ & 0.0001 \\
\hline CD4 count (cells $/ \mathrm{mm}^{3}$ ) & $514(372-712)$ & - & - \\
\hline
\end{tabular}

* In two HIV-uninfected patients, there was insufficient stool to perform an adequate examination for parasites.

† Only including those aged $<60$ years.

¥ Median household income in Yangon is 300,000 Myanmar Kyat (approximately USD200)/month. 


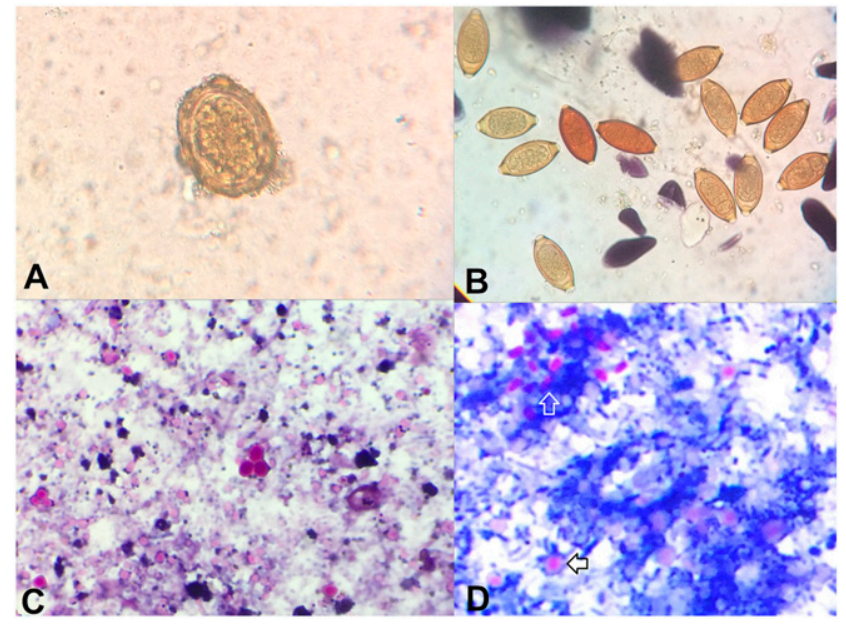

FIGURE 1. Stool microscopy findings in four of the patients. (A) Ascaris lumbricoides ovum. (B) Ova of Trichuris trichiura. (C) Oocysts of Cryptosporidium species. (D) Oocysts of Cryptosporidium (black arrow) and Microsporidium species (white arrow). This figure appears in color at www.ajtmh.org.

Helicobacter pylori infection was more common in the cohort with 124/208 (60\%), having a positive SAT. But HIVinfected patients were no more likely to have a positive SAT than HIV-uninfected patients (63/103 [61\%] versus 61/105
[58\%], $P=0.65)$. Similarly, there was no difference in the demographics, socioeconomic indicators, symptoms, or laboratory findings of the $H$. pylori-positive patients compared with those who were negative.

Cryptosporidium oocysts were seen with acid-fast staining in the stools of $89 / 208(43 \%)$, while Microsporidia oocysts were seen in 37/208 (18\%) (Figure 1). However, most of these patients were asymptomatic and were no significant differences between patients who tested positive for these organisms and those who did not; most notably, there was no difference in HIV status (Supplemental Table 1). There was a single case of $G$. duodenalis in an HIV-uninfected patient. Strongyloides stercoralis, E. histolytica, C. philippinensis, and Isospora, Cyclospora, and Schistosoma species were not identified in any patients.

Despite the study's resource-limited, tropical setting, the prevalence of STH infection was relatively low. More than $76 \%$ of the cohort had previously taken deworming therapy (including $22 \%$ in the past year), suggesting that local awareness of these pathogens is high. In contrast, the prevalence and clinical significance of $H$. pylori infection appear underappreciated: Almost $60 \%$ of this cohort's unselected outpatients tested positive for $H$. pylori, which given the imperfect sensitivity of SAT is likely to underestimate the infection's true burden. However, only one patient in the cohort had ever received $H$. pylori eradication.

TABLE 2

Demographic, socioeconomic, clinical, and laboratory characteristics of the patients, stratified by infection with soil-transmitted helminths and H. pylori

\begin{tabular}{|c|c|c|c|c|c|c|}
\hline Variable & STH positive $(n=22)$ & STH negative $(n=184)$ & $P$-value & H. pylori positive $(n=124)$ & H. pylori negative $(n=84)$ & $P$-value \\
\hline \multicolumn{7}{|l|}{ Demographics } \\
\hline Age (years) & $48(33-57)$ & $48(38-60)$ & 0.35 & $47(36-57)$ & $50(38-66)$ & 0.15 \\
\hline Male gender, $n(\%)$ & $6(27 \%)$ & $75(41 \%)$ & 0.22 & $38(35 \%)$ & $38(45 \%)$ & 0.13 \\
\hline Rural residence & $11(50 \%)$ & 55 (30\%) & 0.06 & 41 (33\%) & 25 (30\%) & 0.66 \\
\hline \multicolumn{7}{|l|}{ Socioeconomic characteristics } \\
\hline $\begin{array}{l}\text { Number of people presently living in } \\
\text { household }\end{array}$ & $4(3-5)$ & $4(3-6)$ & 0.37 & $4(3-5)$ & $4(2-5)$ & 0.16 \\
\hline Number of siblings & $5(4-6)$ & $5(4-7)$ & 0.46 & $5(4-7)$ & $5(3-7)$ & 0.92 \\
\hline Employed* $^{*}$ & $14 / 18(78 \%)$ & $67 / 138(49 \%)$ & 0.02 & $50 / 98(51 \%)$ & $32 / 59(54 \%)$ & 0.70 \\
\hline Completed high school & $5(23 \%)$ & $77(42 \%)$ & 0.08 & $51(41 \%)$ & $31(37 \%)$ & 0.54 \\
\hline Private flushing toilet & $3(14 \%)$ & $30(16 \%)$ & 0.75 & $18(15 \%)$ & $16(19 \%)$ & 0.38 \\
\hline Drinks purified drinking water & $12(55 \%)$ & $124(67 \%)$ & 0.23 & $81(65 \%)$ & 57 (68\%) & 0.70 \\
\hline Municipal water & $5(23 \%)$ & $49(27 \%)$ & 0.69 & $30(24 \%)$ & $25(30 \%)$ & 0.37 \\
\hline Water from underground aquifer (well) & $15(68 \%)$ & $130(71 \%)$ & 0.81 & $90(73 \%)$ & $56(67 \%)$ & 0.36 \\
\hline $\begin{array}{l}\text { Household income }>300,000 \mathrm{MMK} / \\
\text { month† }\end{array}$ & $4(18 \%)$ & $48(26 \%)$ & 0.42 & $35(28 \%)$ & $18(21 \%)$ & 0.27 \\
\hline \multicolumn{7}{|l|}{ Relevant medical history } \\
\hline Ever dewormed & $18(82 \%)$ & $141(77 \%)$ & 0.58 & $94(76 \%)$ & $65(77 \%)$ & 0.79 \\
\hline Dewormed in last year & $6(27 \%)$ & $39(21 \%)$ & 0.51 & $31(25 \%)$ & $14(17 \%)$ & 0.15 \\
\hline Prior H. pylori eradication & 0 & $1(0.5 \%)$ & 1 & 1 & 0 & 1 \\
\hline On antiretroviral therapy & $8 / 9(89 \%)$ & $79 / 94(84 \%)$ & 1 & $54 / 63(86 \%)$ & $33 / 40(83 \%)$ & 0.66 \\
\hline \multicolumn{7}{|l|}{ Current clinical findings } \\
\hline Current dyspepsia & $8(36 \%)$ & $53(29 \%)$ & 0.46 & $33(27 \%)$ & $30(36 \%)$ & 0.16 \\
\hline Current abdominal pain & $2(9 \%)$ & $27(15 \%)$ & 0.48 & $14(11 \%)$ & $15(18 \%)$ & 0.18 \\
\hline Current diarrhea & 0 & $9(5 \%)$ & 0.29 & $5(4 \%)$ & $4(5 \%)$ & 0.80 \\
\hline Current melena & 0 & $1(1 \%)$ & 1 & 0 & 1 & 0.40 \\
\hline $\begin{array}{l}\text { Any gastrointestinal symptoms } \\
\text { currently }\end{array}$ & $9(41 \%)$ & $68(37 \%)$ & 0.72 & $42(34 \%)$ & $37(44 \%)$ & 0.14 \\
\hline Body mass index $\left(\mathrm{kg} / \mathrm{m}^{2}\right)$ & $21.8(20.0-24.2)$ & $22.7(19.8-26.3)$ & 0.35 & $23.1(19.9-27.6)$ & $22.1(20.0-24.7)$ & 0.16 \\
\hline \multicolumn{7}{|l|}{ Laboratory findings } \\
\hline Hemoglobin (g/dL) & $12.1(10.3-12.9)$ & $12.7(11.5-13.6)$ & 0.08 & $12.4(11.5-13.5)$ & $12.6(11.5-13.7)$ & 0.76 \\
\hline Mean corpuscular volume (fL) & $82(74-87)$ & $85(78-90)$ & 0.14 & $84(79-89)$ & $86(78-90)$ & 0.40 \\
\hline HIV positive & $9(41 \%)$ & $94(51 \%)$ & 0.50 & $63(51 \%)$ & $40(48 \%)$ & 0.65 \\
\hline CD4 count (cells $/ \mathrm{mm}^{3}$ ) & 567 (427-902) & 508 (369-709) & 0.30 & $549(403-781)$ & $493(288-650)$ & 0.06 \\
\hline
\end{tabular}

H. pylori $=$ Helicobacter pylori; STH = soil-transmitted helminth. Numbers represent the absolute number (\%) or median (interquartile range).

* Only including those aged $<60$ years.

† Median household income in Yangon is 300,000 Myanmar Kyat (approximately USD200)/month. 
Myanmar has the highest burden of $H$. pylori in Southeast Asia, with one recent study reporting a prevalence of $69 \%{ }^{9}$ Meanwhile, gastric cancer is the country's sixth most common cancer and has a high attributable mortality. ${ }^{10,13} \mathrm{Heli}-$ cobacter pylori is simple to treat, and the risk of recurrence is relatively low. ${ }^{14}$ Indeed, a recent study performed in Yangon showed that local first-line eradication regimens had a per-protocol efficacy that approached $95 \% .{ }^{15} \mathrm{How}-$ ever, the fact that only a single patient in the entire cohort had been previously treated for $H$. pylori suggests that local clinicians are not yet managing the infection as aggressively as they might.

The high prevalence of Cryptosporidium infection was surprising. Equally notable was the fact that there was no difference in the prevalence of the Cryptosporidium between immunocompetent and immunocompromised populations. The absence of symptoms in so many of the patients with Cryptosporidium ova seen in their stools was also noteworthy. Asymptomatic carriage of Cryptosporidium is certainly described, ${ }^{16}$ although not at the rates seen in this series. It is hypothesized that some genotypes of Cryptosporidium may be less pathogenic and, thus, seen more commonly in asymptomatic patients. ${ }^{17}$ However, the absence of molecular testing in the resource-poor setting of the study precluded us from either testing this hypothesis or from confirming or speciating the Cryptosporidium cases diagnosed with modified Ziehl-Neelsen staining. Microsporidia were also common, although again the prevalence was not higher in the HIVinfected patients and most patients were asymptomatic.

The study has many limitations. Gastrointestinal parasites were diagnosed using stool microscopy. Molecular techniques have increased sensitivity and may have assisted with speciation of the Cryptosporidium and Microsporidium cases. ${ }^{7,17,18}$ Only adults attending the outpatients department at a single tertiary referral center were enrolled in the study. Although most of the cohort lived outside Yangonmore than $30 \%$ in a rural location-the results from this small sample are not necessarily representative of the country. Children-particularly in remote locations-are likely to have a higher burden of STH infection. ${ }^{2}$ As antibiotic use in the prior 4 weeks is a contraindication to the use of SAT, this effectively excluded all HIV-infected patients with a CD4 T-cell count of $<350$ cells $/ \mathrm{mm}^{3}$, a population for whom co-trimoxazole is recommended in Myanmar. This led to enrollment of patients with less immunodeficiency, tending to minimize the difference in the array of pathogens identified in the HIV-infected and HIV-uninfected participants. This also limits the generalizability of our findings to the wider local HIV-infected population. ${ }^{4}$ The relatively small sample increases the likelihood of type 2 errors. Future studies should define the relationship between the gastrointestinal pathogens and clinically meaningful endpoints. They should also be powered to determine optimal strategies to identify and treat the infections in a timely and costeffective manner.

Despite this, the findings do offer some insights into the present burden of gastrointestinal pathogens in Myanmar. There appears to be good local awareness of gastrointestinal parasites: most patients had had received deworming therapy previously, many within the previous 12 months. However, $H$. pylori, an infection with more significant health implications, remains relatively neglected in Myanmar, and indeed in low- and middle-income countries generally. ${ }^{19}$ Although there has been progress against many infectious diseases at the national level in Myanmar, ${ }^{20} \mathrm{H}$. pylori has not yet been targeted. These data suggest it is now time to redress this issue.

Received September 30, 2019. Accepted for publication January 13, 2020.

Published online February 24, 2020.

Note: Supplemental table appears at www.ajtmh.org.

Acknowledgments: We would like to thank Naw Zar Aye and Myat Thi Han Theint for performing the stool microscopy. We would like to also acknowledge the support of the doctors and nurses at Insein General Hospital who assisted with the management of the participants.

Disclosures: The stool antigen tests used in the study were provided at a discounted price by BioMérieux.

Authors' addresses: Win Pa Pa Aung and Mya Mya Lwin, Department of Microbiology, University of Medicine 2, Yangon, Myanmar, E-mails: eipamicro@gmail.com and mmlwindr@gmail.com. Nan Phyu Sin Toe Myint, Department of Microbiology, University of Medicine 2, Yangon, Myanmar, and Medicine, Insein General Hospital, Yangon, Myanmar, E-mail: phyujasmine23@gmail.comm. Thet Tun Zaw and Mar Mar Kyi, Medicine, Insein General Hospital, Yangon, Myanmar, E-mails: thattunzaw1995.tz@gmail.com and drmmkyi@gmail.com. David A. Cooper, The Director's Unit, The Kirby Institute, Sydney, Australia, E-mail: dcooper@kirby.unsw.edu.au. Ne Myo Aung, Department of Medicine, University of Medicine 2, Yangon, Myanmar, E-mail: drnaymyoaung@gmail.com. Josh Hanson, The Director's Unit, The Kirby Institute, University of New South Wales, Sydney, Australia, and General Medicine, Cairns Hospital, Cairns, Australia, E-mail: jhanson@kirby.unsw.edu.au.

\section{REFERENCES}

1. Kim MJ, Jung BK, Cho J, Kim DG, Song H, Lee KH, Cho S, Htoon $\mathrm{TT}$, Tin HH, Chai JY, 2016. Prevalence of intestinal Protozoans among schoolchildren in suburban areas near Yangon, Myanmar. Korean J Parasitol 54: 345-348.

2. Silver ZA, Kaliappan SP, Samuel P, Venugopal S, Kang G, Sarkar R, Ajjampur SSR, 2018. Geographical distribution of soil transmitted helminths and the effects of community type in south Asia and south east Asia-a systematic review. PLoS Negl Trop Dis 12: e0006153.

3. UNAIDS estimates, 2018. Country Factsheet: Myanmar. Available at: https://www.unaids.org/en/regionscountries/countries/ myanmar. Accessed September 15, 2019.

4. Aung NM, Hanson J, Kyi TT, Htet ZW, Cooper DA, Boyd MA, Kyi MM, Saw HA, 2017. HIV care in Yangon, Myanmar; successes, challenges and implications for policy. AIDS Res Ther 14: 10.

5. Nsagha DS, Njunda AL, Assob NJC, Ayima CW, Tanue EA, Kibu OD, Kwenti TE, 2016. Intestinal parasitic infections in relation to CD4(+) T cell counts and diarrhea in HIV/AIDS patients with or without antiretroviral therapy in Cameroon. BMC Infect Dis 16: 9.

6. Kaneshiro $Y$ et al., 2019. Intestinal helminth infections in HIVinfected patients in Savannakhet after establishment of an HIV registration network in Lao People's Democratic Republic. Trop Med Health 47: 14.

7. Jourdan PM, Lamberton PHL, Fenwick A, Addiss DG, 2018. Soiltransmitted helminth infections. Lancet 391: 252-265.

8. Means AR, Burns P, Sinclair D, Walson JL, 2016. Antihelminthics in helminth-endemic areas: effects on HIV disease progression. Cochrane Database Syst Rev 4: CD006419.

9. Vilaichone RK, Quach DT, Yamaoka Y, Sugano K, Mahachai V, 2018. Prevalence and pattern of antibiotic resistant strains of Helicobacter pylori infection in ASEAN. Asian Pac J Cancer Prev 19: 1411-1413.

10. World Health Organization, 2017. Age Adjusted Death Rate Estimates: 2017. Available at:https://www.worldlifeexpectancy. com/country-health-profile/myanmar. Accessed June 16, 2019.

11. Nevin DT, Morgan CJ, Graham DY, Genta RM, 2014. Helicobacter pylori gastritis in HIV-infected patients: a review. Helicobacter 19: 323-329. 
12. BioMerieux, 2016. BioNexia H. pylori Package Insert. Marcyl'Etoile, France: bioMérieux.

13. Nwe K, Aung S, Ohnmar M, Mon S, 2016. RM-050 lifestyle habits and dietary patterns in gastric cancer patients in Toungoo, Myanmar. Ann Oncol 27 (Suppl 2): ii97.

14. Xue Y, Zhou LY, Lu HP, Liu JZ, 2019. Recurrence of Helicobacter pylori infection: incidence and influential factors. Chin Med $J$ (Engl) 132: 765-771.

15. Myint N, Zaw TT, Sain K, Waiyan S, Danta M, Cooper D, Aung NM, Kyi MM, Hanson J, 2019. Sequential Helicobacter pylori eradication therapy in Myanmar; a randomized clinical trial of efficacy and tolerability. J Gastroenterol Hepatol. 2019 Nov 23. doi: 10.1111/jgh.14942. [Epub ahead of print]. Available at: https://www.ncbi.nlm.nih.gov/pubmed/31758571.
16. Davies AP, Campbell B, Evans MR, Bone A, Roche A, Chalmers $\mathrm{RM}, 2009$. Asymptomatic carriage of protozoan parasites in children in day care centers in the United Kingdom. Pediatr Infect Dis J 28: 838-840.

17. Davies AP, Chalmers RM, 2009. Cryptosporidiosis. BMJ339: b4168.

18. Anane S, Attouchi H, 2010. Microsporidiosis: epidemiology, clinical data and therapy. Gastroenterol Clin Biol 34: 450-464.

19. Hooi JKY et al., 2017. Global prevalence of Helicobacter pylori infection: systematic review and meta-analysis. Gastroenterology 153: 420-429.

20. Mu TT, Sein AA, Kyi TT, Min M, Aung NM, Anstey NM, Kyaw MP, Soe C, Kyi MM, Hanson J, 2016. Malaria incidence in Myanmar 2005-2014: steady but fragile progress towards elimination. Malar J 15: 503. 